\title{
miR-9 regulates the multidrug resistance of chronic myelogenous leukemia by targeting ABCB1
}

\author{
YAN LI $^{1 *}$, LIFEN ZHAO ${ }^{2 *}$, NANA LI ${ }^{2}$, YUAN MIAO $^{2}$, HUIMIN ZHOU $^{3}$ and LI JIA ${ }^{2}$ \\ ${ }^{1}$ Department of Clinical Laboratory, The First Affiliated Hospital of Dalian Medical University, Dalian, Liaoning 116011; \\ ${ }^{2}$ College of Laboratory Medicine, Dalian Medical University, Dalian, Liaoning 116044; \\ ${ }^{3}$ Department of Microbiology, Dalian Medical University, Dalian, Liaoning 116044, P.R. China
}

Received August 4, 2016; Accepted February 8, 2017

DOI: $10.3892 /$ or.2017.5464

\begin{abstract}
Multidrug resistance (MDR) is commonly correlated with the poor prognosis of chronic myelogenous leukemia. Aberrant expression of microRNAs (miRNAs) plays an important role in the MDR of various types of cancers. MicroRNA-9 (miR-9) has been confirmed to be dysregulated in many types of cancers. However, the relationship between miR-9 and MDR in chronic myelogenous leukemia (CML) remains largely unknown. In the present study, we showed that miR-9-3p (miR-9) was downregulated in adriamycin (ADR)-resistant K562/ADR cells and CML/MDR patients. Overexpression of miR-9 was sufficient to reverse cancer cell resistance to multiple chemotherapeutics in vitro and sensitized tumors to chemotherapy in vivo. Furthermore, we found that ABCB1 is a direct target of miR-9. These findings suggest that miR-9 plays a critical role in MDR in CML by targeting ABCB1. These results provide new evidence of miR-9 as a biomarker for clinical diagnosis and as a potential therapeutic target for CML.
\end{abstract}

\section{Introduction}

Chronic myelogenous leukemia (CML) is a myeloproliferative disorder resulting from the neoplastic transformation of hematopoietic stem cell (1). At present, tyrosine kinase inhibitors are widely used for the treatment of CML. However, cancerous cells frequently develop multidrug resistance (MDR) to chemotherapy agents.

MDR is defined as the resistance of cancer cells to antineoplastic agents that have distinct structures and mechanisms

Correspondence to: Dr Li Jia, College of Laboratory Medicine, Dalian Medical University, 9 Lvshunnan Road, Xiduan, Dalian, Liaoning 116044, P.R. China

E-mail: jiali0386@sina.com

${ }^{*}$ Contributed equally

Key words: miRNA-9, ABCB1, chronic myelogenous leukemia, multidrug resistance of action (2). The specific mechanisms responsible for the MDR phenotype include pharmacokinetic alterations, tumor microenvironmental changes, or cancer cell-specific factors, which include increased drug efflux or decreased drug uptake, drug inactivation, drug target modification or apoptosis evasion (3). ABCB1 (MDR1/P-glycoprotein) is considered to be accountable for the majority of drug efflux in human cancers. P-glycoprotein (P-gp) is a member of the superfamily of ATP-binding cassette (ABC) transporters. P-gp has been reported to be associated with resistance to multiple drugs in various types of cancer, and changes in its expression or function contribute to MDR (4-6).

MicroRNAs (miRNAs) are a class of non-coding RNAs that are 21-25 nucleotides in length and function as regulators of gene expression (7). The dysregulation of miRNA expression has also been detected in CML (8). Loss of miR-203 was detected and overexpression of miR-203 increased sensitivity to imatinib in BaF3-BCR/ABL (T315I) cells (9). miR-9, as an oncogene or tumor-suppressor gene, plays an important role in many types of cancer $(10,11)$. miR-9-3 was found to be downregulated in breast cancers with either high vascular invasion or the presence of lymph node metastasis (12). miR-9-3 could be used as a DNA methylation target in non-small cell lung cancers (13). miR-9-3 activated the NFKB signaling pathway in chronic lymphocytic leukemia by downregulating NF- $\kappa \mathrm{B} 1$ protein (14). However, the effect of miR-9 in MDR of CML remains unclear.

The present study was carried out to investigate the expression levels of miR-9 in K562 and K562/ADR cells and CML patients. In addition, the functional role of miR-9 in the drug resistance of CML was identified. Moreover, we explored the correlation between the expression of miR-9 and ABCB1.

\section{Materials and methods}

Cell culture. Human chronic myelogenous leukemia blast K562 cells were cultured in RPMI-1640 medium, supplemented with $10 \%$ fetal bovine serum (FBS) and $1 \%$ penicillin-streptomycin (all from Gibco, Grand Island, NY, USA) at $37^{\circ} \mathrm{C}$ in humidified atmosphere containing $5 \% \mathrm{CO}_{2}$. Adriamycin (Sigma, St. Louis, MO, USA) was added to parental cell cultures in stepwise increasing concentrations from 0.001 to $1 \mathrm{mg} / 1$ for 6 months to develop an adriamycin (ADR)-resistant cell line, named 
K562/ADR. For K562/ADR cells, $1 \mathrm{mg} / \mathrm{l}$ of adriamycin was added in daily culture and removed before the experiments.

Primary patient samples. Sixty-one patients were recruited from July 2012 to June 2015 at The First Affiliated Hospital of Dalian Medical University (Dalian, China). The age of patients ranged from 17 to 70 years of age, with a median age of 43. The diagnosis of CML was based on cytomorphology, cytochemistry, multiparameter flow cytometry (FCM), immunology, molecular genetics and cytogenetics. Bone marrow cells were extracted after the patients were admitted to the hospital before treatment. Murine anti-human P-gp (UIC2 clone)-PE was purchased from BioLegend (San Diego, CA, USA). Then, the expression of P-gp was detected by FCM. Peripheral blood mononuclear cells (PBMCs) were isolated using Ficoll-Hypaque and were further cultured in plastic dishes to remove adherent cells at $37^{\circ} \mathrm{C}$ for $24 \mathrm{~h}$. The research protocol was approved by the Ethics Committee of Dalian Medical University. The patient clinical characteristics are shown in Table I.

MicroRNA array. miRNA arrays were performed for K562 and K562/ADR cell groups ( $\mathrm{n}=3$ /group) by Exiqon (KangChen, China) using the miRCURY ${ }^{\mathrm{TM}} \mathrm{Hy} 3^{\mathrm{TM}} / \mathrm{Hy} 5^{\mathrm{TM}}$ power labeling kit and the miRCURY ${ }^{\text {TM }}$ LNA Array (version 10.0; 757 human miRs).

Real-time PCR. Total RNA was isolated from the cell lines with the RNeasy Mini kit, and cDNA was synthesized using QuantiTect Reverse Transcription kit (both from Qiagen, Valencia, CA, USA) according to the manufacturer's protocol. The expression of miR-9 was determined using mirVana ${ }^{\mathrm{TM}}$ qRT-PCR MicroRNA Detection kit according to the manufacturer's protocol (Ambion Inc., Austin, TX, USA) and was normalized to U6-small nuclear RNA. qRT-PCR was performed to detect ABCB1 mRNA using the SYBR-Green PCR kit (Takara Bio, Inc., Otsu, Japan). The sequences of the upstream and downstream primers were as follows: 5'-CCC ATCATTGCAATAGCAGG-3' and 5'-GTTCAAACTTCTG CTCCTGA-3' for ABCB1; 5'-CTCCCTCCACCTTTGA CGCTG-3' and 5'-TCCTCTTGTGCTCTTGCTGG-3' for GAPDH. The expression level of target genes was determined relatively to GAPDH and calculated as: $2^{-\left(\mathrm{Ct}_{\text {Target gene }}-\mathrm{Ct}_{\mathrm{GAPDH}}\right)}$.

Oligonucleotide construction. shRNA specific for ABCB1 (5'-GUUUGUCUACAGUUCGUAA-3'); mimics (5'-AUAA AGCUAGAUAACCGAAAGU-3' and 5'-UUUCGGUUAUCU AGCUUUAUUU-3'); mimic negative control (NC) (5'-UUC UUCGAACGUGUCACGUTT-3' and 5'-ACGUGACACGUU CGGAGAATT-3'); antagomiR (5'-ACUUUCGGUUAUCU AGCUUUAU-3'); and control (5'-CAGUACUUUUGUGUAG UACAA-3') for hsa-miR-9 were obtained from RiboBio Co. Ltd. (Guangzhou, Guangdong, China).

In vitro drug sensitivity assay. Drug resistance was evaluated by MTT assay as previously described (15). Briefly, cells $\left(1 \times 10^{4}\right)$ were plated in a 96-well plate and incubated with different anticancer drugs at varying concentrations (doxorubicin, 0,10 , 30,50 and $100 \mathrm{mg} / \mathrm{l}$; vincristine, $0,10,30,50$ and $100 \mathrm{mg} / \mathrm{l}$; paclitaxel, 0, 1, 5, 20 and $40 \mathrm{mg} / \mathrm{l}$; Sigma), respectively. After
Table I. Clinicopathological characteristics of the CML patients.

\begin{tabular}{lc}
\hline Patient demographics & CML (n=61) \\
\hline Gender & 38 \\
Male & 23 \\
Female & \\
Age (years) & 43 \\
Median & $17-70$ \\
Range & 52 \\
Splenic enlargement & 19 \\
Hemoglobin $<100.0 \mathrm{~g} / 1$ & \\
WBC count $\left(10^{9} / 1\right)$ & 36 \\
$20-100$ & 25 \\
$>100$ & \\
Platelet count $\left(10^{9} / 1\right)$ & 27 \\
$<300$ & 34 \\
$>300$ & 33 \\
P-gp $(+)$ & \\
\hline
\end{tabular}

CML, chronic myelocytic leukemia; P-gp, P-glycoprotein; WBC, white blood cell.

48 h, $100 \mu \mathrm{l}$ MTT (5 mg/l; Sigma) was added to each well and cultured for an additional $4 \mathrm{~h}$. Then, $100 \mu \mathrm{l}$ of dimethyl sulfoxide (DMSO) was added into each well and the absorbance was determined at $490 \mathrm{~nm}$ using a microplate reader (Model550; Bio-Rad Laboratories, Hercules, CA, USA).

Intracellular ADR concentration assays. Fluorescence intensity of intracellular ADR (doxorubicin) was determined using FCM. Briefly, cells were seeded into 6-well plates $\left(1 \times 10^{6}\right.$ cells/ well) and cultured overnight. After addition of ADR to a final concentration of $5 \mu \mathrm{g} / \mathrm{ml}$, the cells continued to be cultured for $1 \mathrm{~h}$. The cells were then harvested. Then, the cells were washed with phosphate-buffered-saline (PBS), and the mean fluorescence intensity of intracellular ADR was detected using FCM. The experiment was independently performed 3 times.

Apoptosis analysis. Cells were seeded onto a 6-well plate at a density of $\sim 2 \times 10^{5}$ cells/well. Following treatment with drugs [(K562/ADR, vincristine/paclitaxel $40 \mu \mathrm{g} / \mathrm{ml}$; K562, vincristine/paclitaxel $1 \mu \mathrm{g} / \mathrm{ml}$ ) (for $48 \mathrm{~h}$ )], the cells were collected, and washed with ice-cold PBS twice. Cells were resuspended in $100 \mu \mathrm{l}$ binding buffer and stained with Annexin V/FITC followed by propidium iodide (PI). Cells were then incubated in the dark for $15 \mathrm{~min}$ at room temperature, and $400 \mu \mathrm{l}$ binding buffer was added. The cells were immediately measured by FACSCalibur (Becton-Dickinson, San Jose, CA, USA).

Luciferase assay. A pmirGLO dual-luciferase miRNA target expression vector was used for the 3'-UTR luciferase assays (Promega, Madison, WI, USA). Plasmids containing wild-type pmirGLO-ABCB1-3'-UTR, mutant pmirGLO-ABCB1-3'-UTR were synthesized. HEK 293T cells 
A

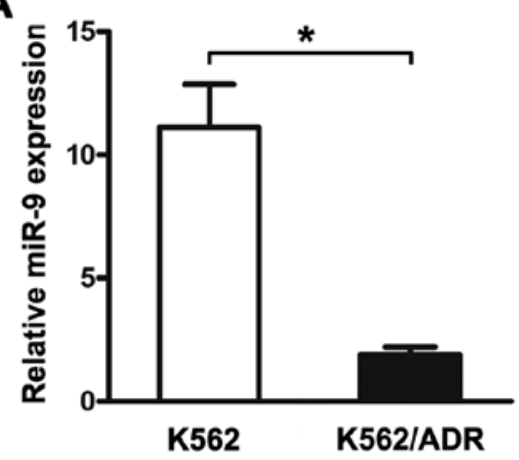

B

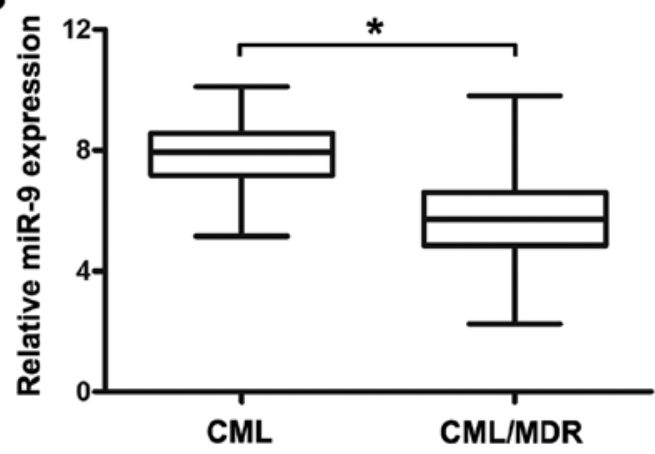

Figure 1. Differential expression of miR-9 in K562 and adriamycin (ADR)-resistant K562/ADR cells and CML patients. (A) The expression of miR-9 was analyzed in K562 and K562/ADR cells. (B) The expression of miR-9 was analyzed in CML and CML/MDR patients. Data are the means \pm SD of triplicate determinants. ${ }^{*} \mathrm{P}<0.05$. CML/MDR, multidrug-resistant chronic myelocytic leukemia.

were seeded $\left(5 \times 10^{4}\right.$ cells/well) in a 24 -well dish and were incubated overnight. For the 3'-UTR luciferase assay, the cells were co-transfected with hsa-miR-9 mimics and wild-type or mutant target sequence using Lipofectamine 2000. The lysates were collected $48 \mathrm{~h}$ after the transfection, and the activities of firefly and Renilla luciferases were measured using the Dual-Luciferase ${ }^{\circledR}$ Reporter Assay system (Promega) and normalized to those of Renilla luciferase activities. The mean of the results from the cells transfected with the miR-control was set at 1.0. Data are presented as the mean value \pm SD for triplicate experiments.

Western blot analysis. The total cell proteins were separated by SDS polyacrylamide gel electrophoresis with $6 \%$ spacer and $10 \%$ separation gels. The protein samples were removed onto polyvinylidene difluoride (PVDF) membranes and blocked with 5\% powdered skimmed milk prepared with TTBS. Then, the PVDF membranes were respectively incubated with P-gp antibody (1/1,000 dilution; Abcam, Cambridge, UK), and then with peroxidase-conjugated anti-rabbit IgG (1/10,000 dilution; GE Healthcare UK Ltd., Little Chalfont, UK). The control was the GAPDH antibody (1/2500 dilution; Bioworld, St. Louis Park, MN, USA). All bands were detected using ECL Western Blot kit (Amersham Biosciences, Buckinghamshire, UK), and analyzed using LabWorks ${ }^{\mathrm{TM}}$ (version 4.6; UVP; BioImaging Systems).

In vivo antitumor activity. Four-week-old male athymic nude mice were obtained from the Animal Facility of Dalian Medical University, and were fed with sterilized food and water. Approximately, $1 \times 10^{7}$ cells (K562/ADR-NC and K562/ADR-miR-9) were subcutaneously injected into the right flank of each nude mouse, respectively. Mice bearing palpable tumors $(\sim 1$ week after tumor cell inoculation) were randomly divided into control and treatment groups (6 animals/group). The mice in groups were intratumorally injected with $100 \mu 1$ mimics or NC combined with an intraperitoneal injection of adriamycin $(7 \mathrm{mg} / \mathrm{kg})$ or PBS 3 times/week for 3 weeks. The mice were sacrificed and their tumors were isolated, weighed and photographed. The tumor volume was calculated by the following formula: Tumor volume $=1 / 2\left(\right.$ length $\mathrm{x}$ width ${ }^{2}$ ) Tumors were removed, weighed and fixed in $4 \%$ paraformaldehyde. These experiments were approved by the Ethics
Committee of the Animal Experiments of the Dalian Medical University.

Immunohistochemical (IHC) staining analysis. Visible tumors were removed from the mice and immunohistochemistry was performed on paraffin-embedded sections. The slides were dried, deparaffinized and rehydrated. After deparaffinization and blocking of endogenous peroxidase, the slides were labeled overnight at $4^{\circ} \mathrm{C}$ with antibodies (Abcam) at a dilution of 1:200. The following staining was performed at room temperature for 60 min with secondary streptavidin-HRP-conjugated antibody (Santa Cruz Biotechnology, Inc., Santa Cruz, CA, USA). Finally, the sections were counterstained with hematoxylin and coverslipped. The Image-Pro Plus 4.5 software (Media Cybernetics, Inc., Rockville, MD, USA) was used to analyze the expression of proteins.

Statistical analysis. Each test was performed at least in triplicate, and the data are expressed as means \pm standard deviation (SD). Student's t-test was used to compare the means of two groups. $\mathrm{P}<0.05$ was considered to indicate a statistically significant result. All analyses were performed using SPSS 13.0 statistical packages (SPSS, Inc., Chicago, IL, USA).

\section{Results}

Differential expression of miR-9 in K562 and K562/ADR cells and CML patients. Real-time PCR was used to analyze the expression of miR-9 in the K562 and K562/ADR cells and CML patients. As shown in Fig. 1A, the expression of miR-9 was obviously increased in the K562 cells, compared with that noted in the K562/ADR cells.

To further investigate the expression of miR-9 in CML patients, the PBMCs isolated from CML patients were analyzed by qRT-PCR. The PBMCs were first divided into two groups, CML without MDR and CML/MDR. The frequency of P-gp positivity was $54.1 \%$ (33 of 61 ) in the CML patients. It was shown that miR-9 expression was significantly lower in the CML/MDR patients compared to that noted in the CML patients (Fig. 1B). The data indicated that differential expression of miR-9 may be related to the drug-resistance of human CML. 
A

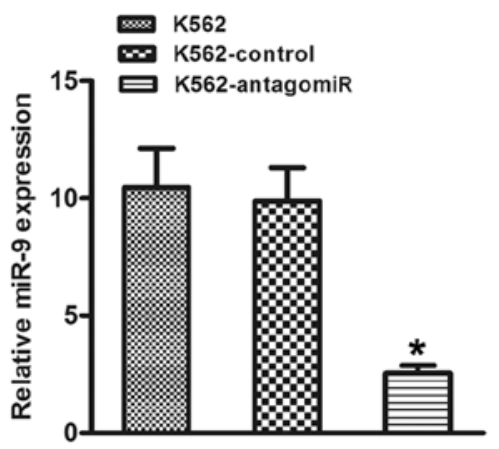

B $\mathrm{K} 562$

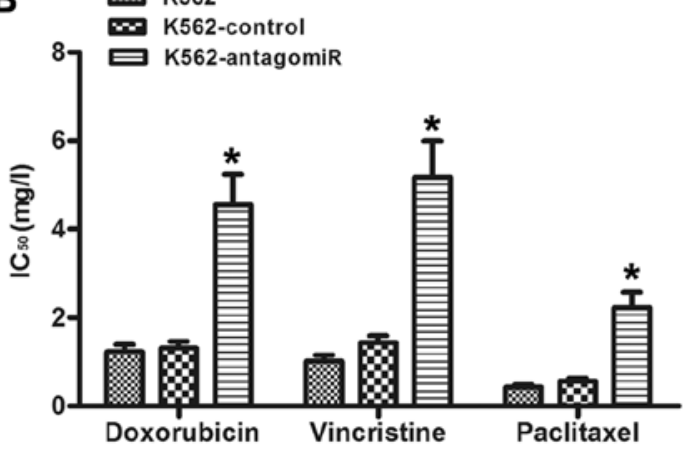

C
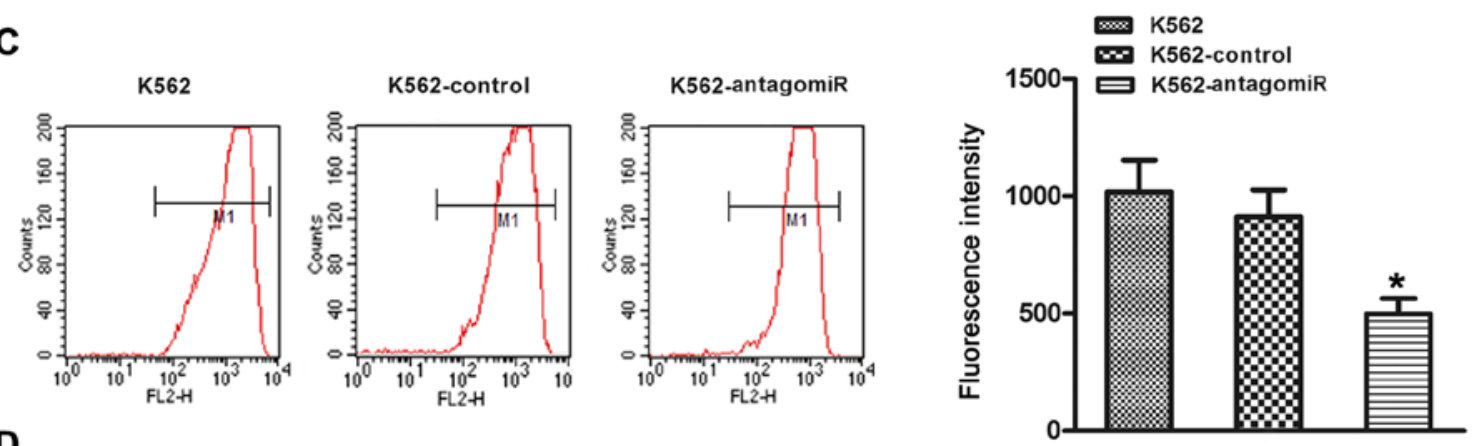

D
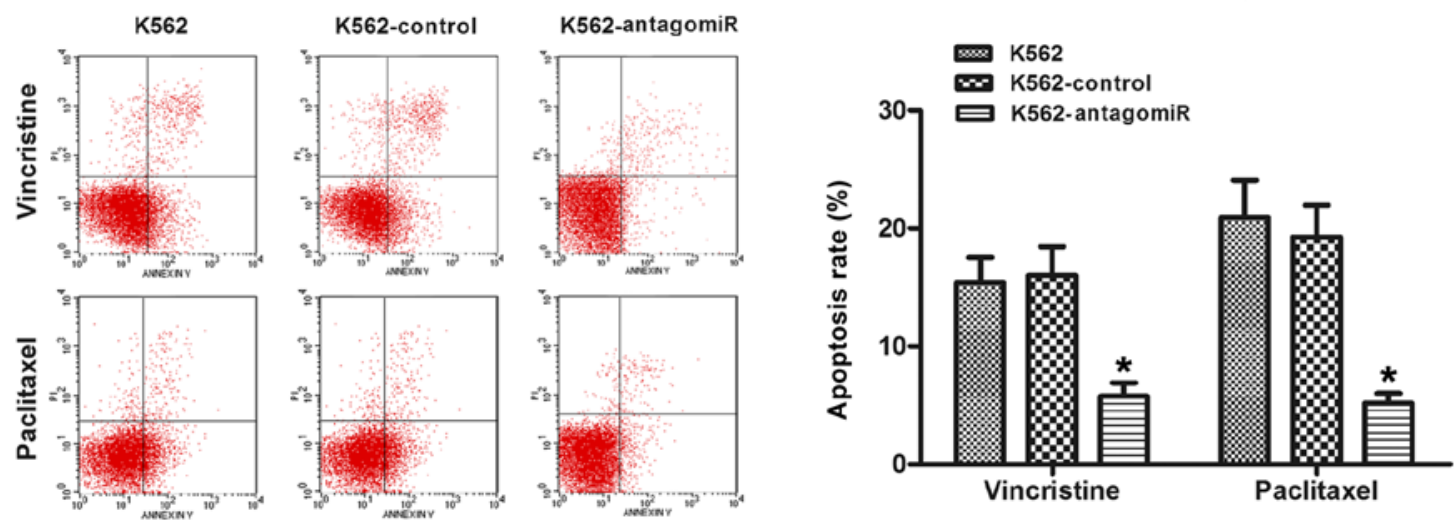

Figure 2. Inhibition of miR-9 decreases the chemosensitivity of K562 cells in vitro. (A) The expression of miR-9 was analyzed in K562 cells transfected with the antagomiR. (B) Cell chemosensitivity was assessed by MTT assays. (C) The intracellular adriamycin (ADR) accumulation was measured. (D) The apoptotic rates of cells treated with vincristine or paclitaxel were measured by flow cytometry. The data represent the means \pm SD of 3 independent assays $($ ( $\mathrm{P}<0.05)$.

Inhibition of miR-9 decreases the chemosensitivity of K562 cells in vitro. In order to investigate the effect of miR-9 on the chemosensitivity of CML cells, the K562 cells were transfected with miR-9 antagomiR. As shown in Fig. 2A, the expression of miR-9 was obviously decreased compared with the control group. The $\mathrm{IC}_{50}$ values (drug concentration that inhibits cell growth by $50 \%$ ) were significantly increased in the K562 cells transfected with the miR-9 antagomiR (Fig. 2B), compared to the control. Since MDR of cancer is mainly due to alterations of drug influx and efflux, ADR was used as a probe to evaluate drug accumulation in the cancer cells. Thus, ADR intracellular accumulation was explored. As shown in Fig. 2C, decreased accumulation of ADR was observed in the K562-antagomiR cells compared with the control cells $(\mathrm{P}<0.05)$. Then, the role of miR-9 downregulation in the survival of drug-resistant cells was investigated. Annexin V assays demonstrated that silencing of miR-9 reduced the apoptosis rate of K562 cells by resistance to the chemotherapeutic agent vincristine or paclitaxel (Fig. 2D).
Overexpression of miR-9 increases the chemosensitivity of K562/ADR cells in vitro. In order to further study the effect of miR-9 on chemosensitivity, the K562/ADR cells were transfected with miR-9 mimics. As shown in Fig. 3A, the expression level of miR-9 was increased in the K562/ADR-mimic cells compared with the NC cells. In addition, overexpression of miR-9 sensitized cells to chemotherapy, as indicated by a decrease in the $\mathrm{IC}_{50}$ values (Fig. 3B). Moreover, the restoration of miR-9 increased the level of intracellular ADR (Fig. 3C). Meanwhile, ectopic expression of miR-9 increased the apoptosis rate of K562/ADR cells treated with chemotherapeutic agent vincristine or paclitaxel (Fig. 3D).

$A B C B 1$ is a target of miR-9. Bioinformatic analyses suggested that $A B C B 1$ is a potential target of miR-9. To test whether $\mathrm{ABCB} 1$ is a direct target of miR-9, luciferase activity assays were performed. 293T cells were transfected with luciferase reporters carrying the ABCB1-wt-3'-UTR or ABCB1-mut3'-UTR, along with mimics or NC. As shown in Fig. 4A, 
A

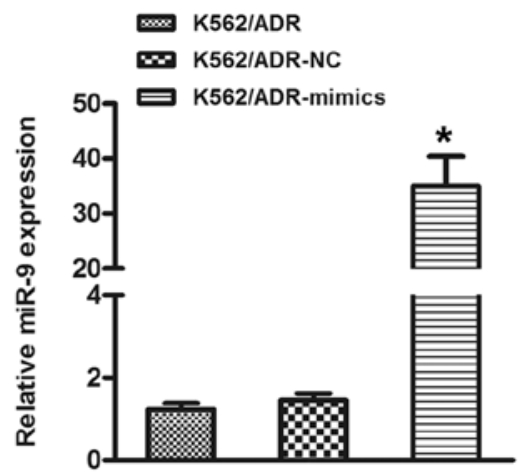

B $\quad$ K562/ADR

K562/ADR-NC

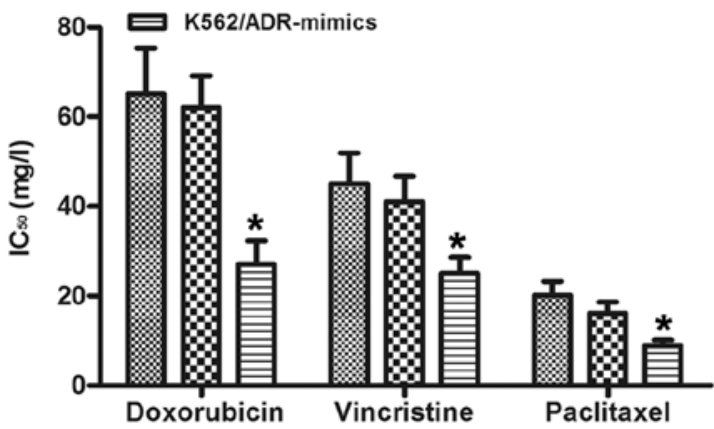

C

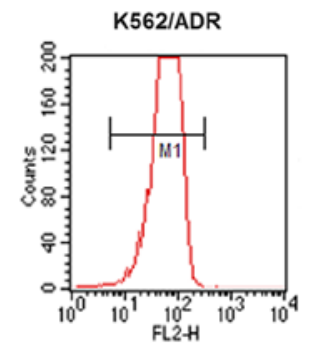

D
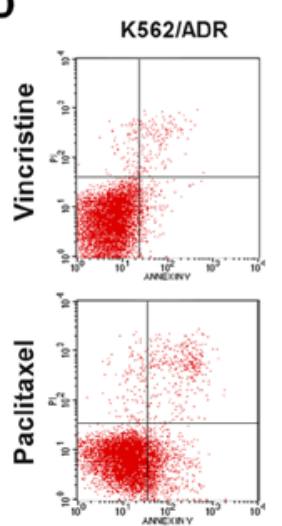

K562/ADR-NC

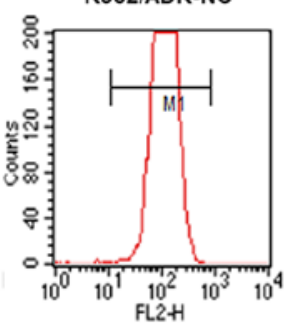

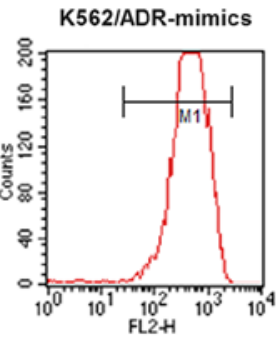
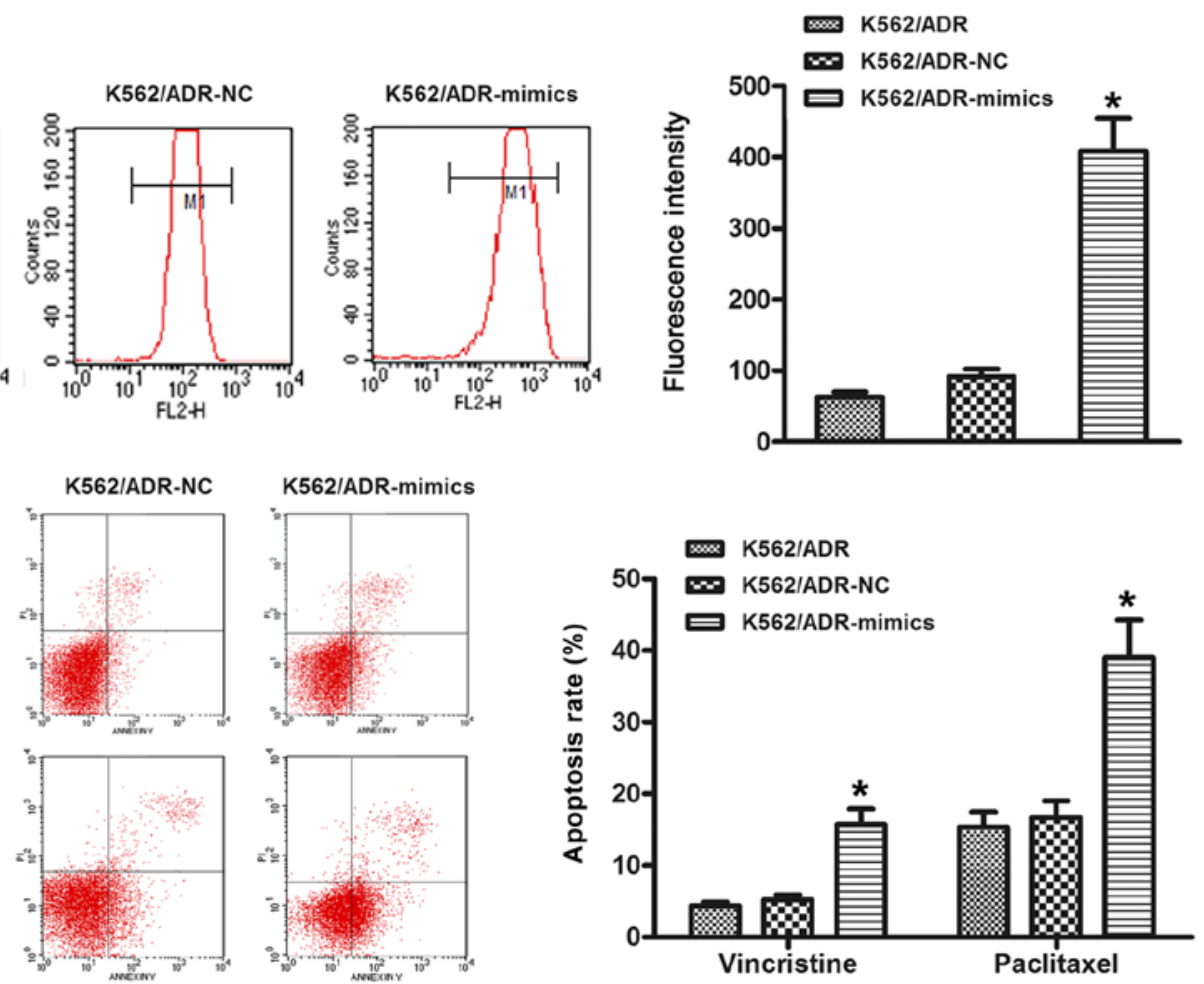

Figure 3. Overexpression of the miR-9 increases the chemosensitivity of adriamycin (ADR)-resistant K562/ADR cells in vitro. (A) The expression of miR-9 was confirmed in cells transfected with the mimics. (B) The $\mathrm{IC}_{50}$ values of K562/ADR cells to doxorubicin, vincristine and paclitaxel were measured ("P $<0.05$ ). (C) Fluorescence intensity analysis of intracellular ADR. (D) The apoptotic rates of cells treated with vincristine or paclitaxel were detected by flow cytometry. The data represent the means \pm SD of 3 independent assays ( $\mathrm{P}<0.05)$.

forced miR-9 expression decreased luciferase activity, and this suppression was reversed by the mutation of the target sequences in the 3'-UTRs of ABCB1. Furthermore, the expression of ABCB1 mRNA was analyzed by RT-PCR and the expression of P-gp proteins was analyzed by western blotting. The results showed that the ABCB1 mRNA and P-gp protein were obviously increased after the knockdown of miR-9 in the K562 cells (Fig. 4C). Conversely, the ABCB1 mRNA and P-gp protein were significantly decreased after the overexpression of miR-9 in the K562/ADR cells (Fig. 4D). Moreover, P-gp expression in CML patients was detected by FCM. As shown in Fig. 4B, the expression levels of miR-9 were negatively correlated with P-gp expression in the CML patients $(\mathrm{r}=-0.6922, \mathrm{P}<0.0001)$.

$A B C B 1$ reverses the effect of miR-9-mediated chemoresistance in $\mathrm{K} 562$ cells. To further confirm that ABCB1 is a direct target of miR-9, we performed a rescue experiment by co-transfecting ABCB1 shRNA (vs. the shRNA control) and miR-9 antagomiR (vs. the control) into K562 cells. Transfection of miR-9 antagomiR led to the upregulation of P-gp protein as determined by western blot analysis. Importantly, the upregulation of P-gp induced by miR-9 antagomiR was effectively reversed by ABCB1 shRNA (Fig. 5A). Moreover, the increase in drug resistance induced by miR- 9 antagomiR was attenuated by ABCB1 shRNA (Fig. 5B).

Altered expression of miR-9 influences the chemosensitivity of K562/ADR cells in vivo. Then, we investigated whether miR-9 regulates the chemosensitivity of CML cells in vivo. As shown in Fig. 6A, overexpression of miR-9 in K562/ADR cells resulted in a significantly lower tumor volume compared with the cells treated with $\mathrm{NC}$ after 3 weeks of ADR treatment. In addition, the tumor volume in the K562/ADR-miR-9 group was markedly 
A

wt-ABCB1 5' UagauaAcuUcugcugcuUUaA 3' miR-9 3' UGAAAGCCAAUAGAUCGAIII miR-9 3' UGAAAGCCAAUAGAUCGAAAUA 5'
mut-ABCB1 5' UAGAUAACUUCUGCUGUGCGÁ 3'

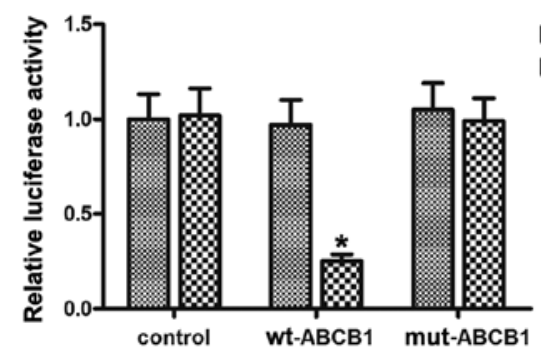

C

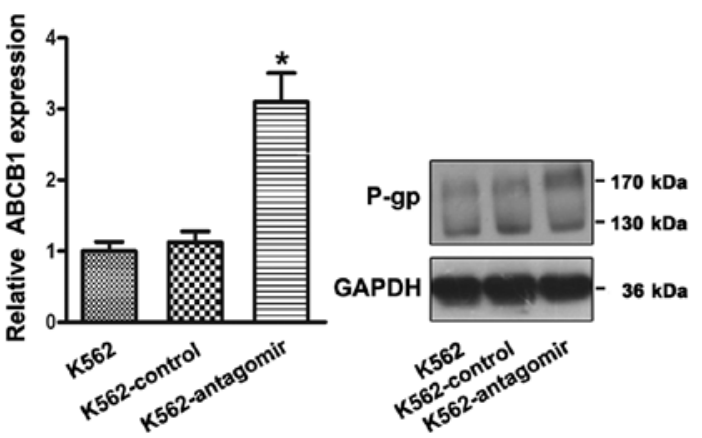

B

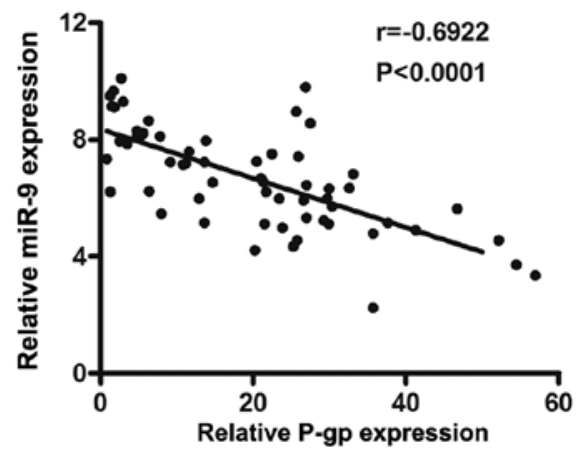

D

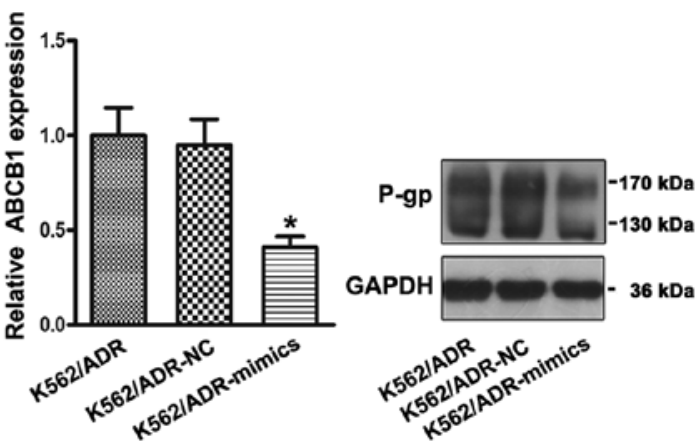

Figure 4. ABCB1 is a target of miR-9. (A) The wild-type and mutant miR-9 target sequences of ABCB1 3'-UTR (upper panel); luciferase assay for the direct targeting of wt-3'-UTR of ABCB1 by miR-9 (lower panel). (B) Correlation between miR-9 and P-glycoprotein (P-gp) expression levels in CML patients ( $\mathrm{n}=61$ ). Each point in this scatter graph represents an individual sample, in which the relative miR-9 level is indicated on the $y$-axis and the P-gp level on the $\mathrm{x}$-axis. The y-axis indicates normalized expression of miR-9 as examined by quantitative RT-PCR. The x-axis indicates expression of P-gp as determined using flow cytometry. (C) Relative ABCB1 mRNA expression and P-gp proteins were analyzed by RT-PCR and western blotting after the knockdown of miR-9 in K562 cells. (D) Relative ABCB1 mRNA expression and P-gp protein were analyzed by RT-PCR and western blotting after the overexpression of miR-9 in adriamycin (ADR)-resistant K562/ADR cells. The data represent the means \pm SD of 3 independent assays ( $\mathrm{P}<0.05$ ). CML, chronic myelocytic leukemia.

A

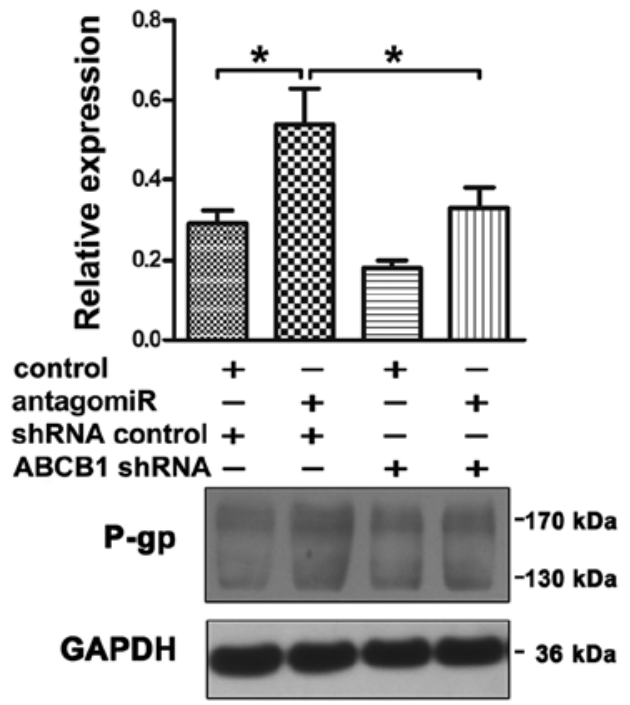

B

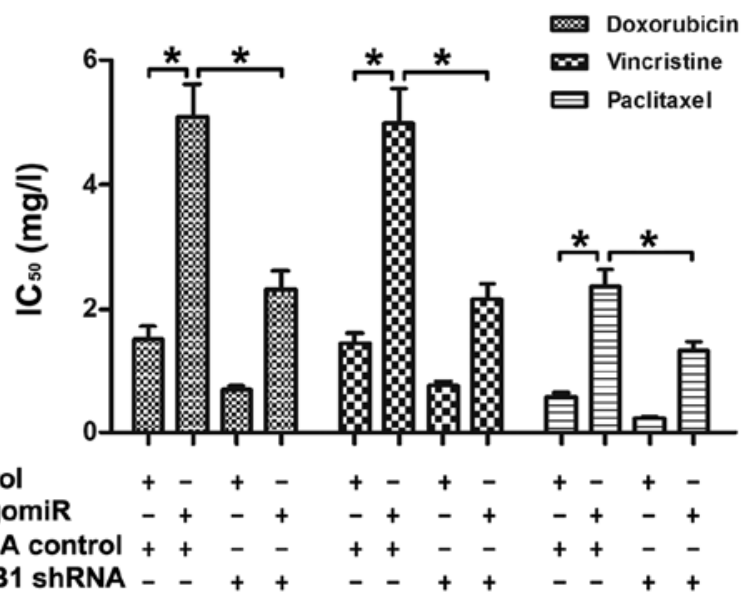

Figure 5. ABCB1 reverses the effect of miR-9-mediated chemoresistance in K562 cells. (A) Relative expression of P-glycoprotein (P-gp) was analyzed by western blotting. K562 cells were co-transfected with antagomiR and shRNAs targeting ABCB1. Expression of the P-gp protein was detected by western blotting. (B) Cell chemosensitivity was assessed by MTT assays. Data are the means \pm SD of triplicate determinants $($ ( $P<0.05)$.

decreased compared with that in the K562/ADR-NC group. At the end of this experiment, the mice were sacrificed and their tumors were isolated. We further analyzed the P-gp expression pattern in the tumors by IHC staining. The expression of P-gp 
A
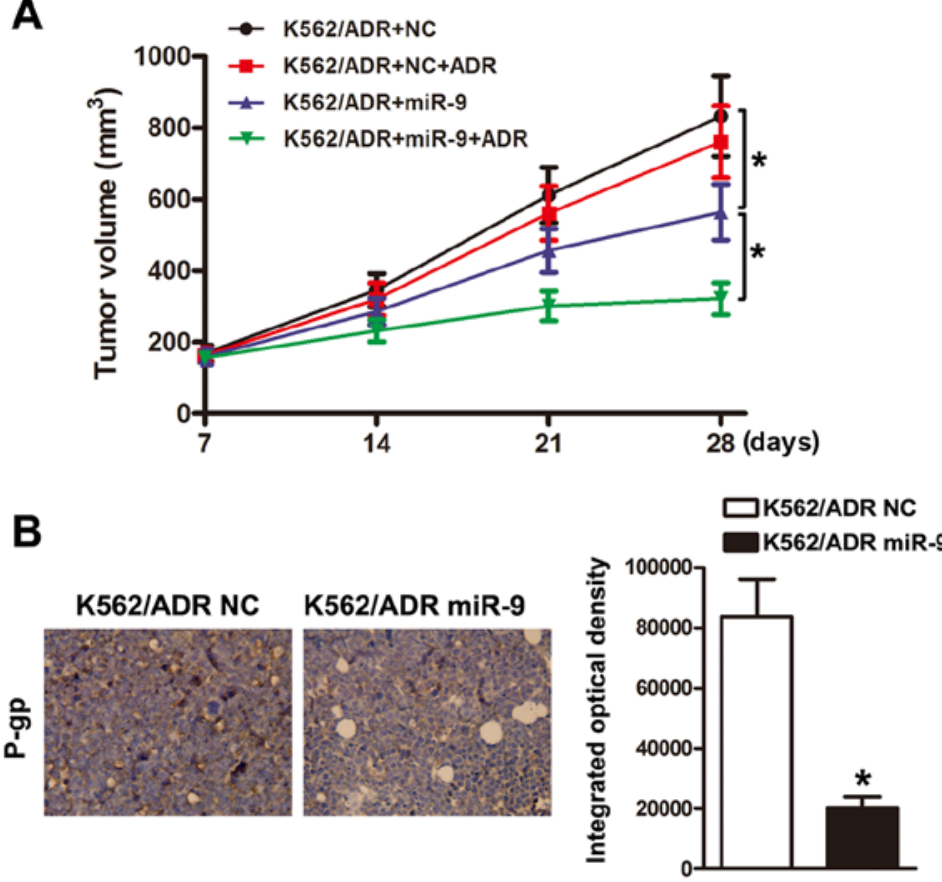

Figure 6. Altered expression of miR-9 influences the chemosensitivity of adriamycin (ADR)-resistant K562/ADR cells in vivo. (A) The tumor volumes were measured at the indicated time points. (B) The expression level of P-glycoprotein (P-gp) was determined by IHC staining in tumor tissues. The data were the mean $\pm \mathrm{SD}$ of 3 separate transfections $\left({ }^{*} \mathrm{P}<0.05\right)$

was decreased in the K562/ADR-miR-9 group compared with the expression noted in the NC group (Fig. 6B). These results were consistent with the results in vitro.

\section{Discussion}

Multidrug resistance (MDR) and disease relapse is a challenging clinical issue in the treatment of leukemia. In the present study, we found that the expression of miR-9 was obviously increased in the K562 cells and CML patients. It was shown that miR-9 regulated the CML drug resistance both in vitro and in vivo. In addition, we found that miR-9 and P-glycoprotein (P-gp) had a negative correlation in the CML patients. Furthermore, we found that $\mathrm{ABCB} 1$ is a direct target of miR-9.

miRNAs plays an important role in the chemoresistance of various types of cancers, by targeting the 3 '-untranslated region (3'-UTR) of a specific mRNA via base pairing. miR-181b has been reported to be involved in MDR in gastric cancer (15). miR-221/-222 were found to increase ER-independent growth and confer resistance to fulvestrant by the activation of $\beta$-catenin in breast cancer (16). It was reported that miR-214 negatively regulates phosphatase and tensin homolog (PTEN), which is a tumor suppressor, leading to induced cisplatin resistance (17).

Several studies have described regulatory functions for miRNAs in the chemoresponsiveness of leukemia cells. Overexpression of miR-370 sensitized K562 cells to homoharringtonine (18). Moreover, miR-17 and miR-20a have been reported to be involved in the chemoresistance of leukemia cells (19). Additionally, ectopic expression of miR-217 was found to sensitize dasatinib-resistant K562 cells to dasatinib (20). More recently, downregulation of miR-21 increased apoptosis in CML (21). However, the role of miRNAs in the drug resistance of leukemia has not been fully addressed. In the present study, we found that the expression of miR-9 was obviously increased in K562 cells, compared with that in the K562/ADR cells. Moreover, miR-9 expression was significantly lower in CML/MDR patients compared to CML patients. Inhibition of miR-9 decreased the chemosensitivity of K562 cells in vitro. Moreover, overexpression of miR-9 increased the chemosensitivity of K562/ ADR cells in vitro and in vivo. Notably, the tumor volumes in the K562/ADR-miR-9 group were markedly decreased compared with tumor volumes in the K562/ADR-NC group. The potential reason may be that miRNA has hundreds or thousands of mRNA targets; a broad segment of the protein coding genome is under their control. It has been reported that high miR-9 expression significantly sensitized ovarian cancer cells to cisplatin in vivo and in vitro (22), consistent with the present study. However, another study demonstrated that miR-9 promoted the chemoresistance of bladder cancer cells by targeting LASS2 (23). This may suggest that miR-9 is involved in the response to treatment. The role of miR-9 as an oncogene or as a tumor suppressor for tumor chemoresistance depends on the cell type.

$\mathrm{P}-\mathrm{gp}$ is a membrane transporter glycoprotein. The overexpression of $\mathrm{P}$-gp in cancer cells promotes drug resistance (6), whereas inhibition of the expression or function of P-gp reverses drug resistance (3). miR-451 was found to be inversely correlated with MDR1 expression in drug-resistant breast cancer cells (24). It was also reported that upregulated miR-27a and miR-451 stimulated MDR1 expression in cell lines of human ovarian cancer and cervix carcinoma (25). More 
recently, miR-331-5p and miR-27a were found to regulate P-gp by directly binding to its 3'-UTR (26). In the present study, it was confirmed that ABCB1 is a direct target of miR-9 in CML cells by luciferase activity analysis. An altered level of miR-9 led to a change in ABCB1 mRNA and P-gp proteins. Then, we detected the expression of miR-9 and P-gp in CML patients. It was found that miR-9 and P-gp had a negative correlation. Moreover, silencing of ABCB1 reversed the drug resistance induced by the miR- 9 antigomir. Furthermore, miR-9 inhibited the expression of P-gp protein in tumor tissues. These observations indicate that miR-9 may serve as an MDR-specific miRNA in human CML by targeting ABCB1.

In summary, miR-9 regulated the drug resistance of CML. miR-9 may be a potential therapeutic target for the treatment of the MDR of CML in the future. Certainly, there are additional target genes that contribute to the function of miR-9 in CML. Therefore, further effort is warranted to expand the miR-9 downstream functional network.

\section{Acknowledgements}

The present study was supported by grants from the National Natural Science Foundation of China (no. 81472014).

\section{References}

1. Sloma I, Jiang X, Eaves AC and Eaves CJ: Insights into the stem cells of chronic myeloid leukemia. Leukemia 24: 1823-1833, 2010.

2. Fojo A, Hamilton TC, Young RC and Ozols RF: Multidrug resistance in ovarian cancer. Cancer 60 (Suppl 8): S2075-S2080, 1987.

3. Zinzi L, Capparelli E, Cantore M, Contino M, Leopoldo M and Colabufo NA: Small and innovative molecules as new strategy to revert MDR. Front Oncol 4: 2, 2014.

4. Hendrikx JJ, Lagas JS, Rosing H, Schellens JH, Beijnen JH and Schinkel AH: P-glycoprotein and cytochrome P450 3A act together in restricting the oral bioavailability of paclitaxel. Int J Cancer 132: 2439-2447, 2013.

5. Zhu H, Liu Z, Tang L, Liu J, Zhou M, Xie F, Wang Z, Wang Y, Shen S, Hu L, et al: Reversal of P-gp and MRP1-mediated multidrug resistance by $\mathrm{H} 6$, a gypenoside aglycon from Gynostemma pentaphyllum, in vincristine-resistant human oral cancer (KB/VCR) cells. Eur J Pharmacol 696: 43-53, 2012.

6. Tsuji K, Wang YH, Takanashi M, Odajima T,Lee GA, Sugimori H and Motoji T: Overexpression of lung resistance-related protein and P-glycoprotein and response to induction chemotherapy in acute myelogenous leukemia. Hematol Rep 4: e18, 2012.

7. Zhang B, Pan X, Cobb GP and Anderson TA: microRNAs as oncogenes and tumor suppressors. Dev Biol 302: 1-12, 2007.

8. Wang LS, Li L, Li L, Chu S, Shiang KD, Li M, Sun HY, Xu J, Xiao FJ, Sun G, et al: MicroRNA-486 regulates normal erythropoiesis and enhances growth and modulates drug response in CML progenitors. Blood 125: 1302-1313, 2015.

9. Li Y, Yuan Y, Tao K, Wang X, Xiao Q, Huang Z, Zhong L, Cao W, Wen $J$ and Feng W: Inhibition of BCR/ABL protein expression by miR-203 sensitizes for imatinib mesylate. PLoS One 8: e61858, 2013.

10. Tsai KW, Liao YL, Wu CW, Hu LY, Li SC, Chan WC, Ho MR, Lai CH, Kao HW, Fang WL, et al: Aberrant hypermethylation of $m i R-9$ genes in gastric cancer. Epigenetics 6: 1189-1197, 2011.
11. Zhang H, Qi M, Li S, Qi T, Mei H, Huang K, Zheng L and Tong Q: microRNA-9 targets matrix metalloproteinase 14 to inhibit invasion, metastasis, and angiogenesis of neuroblastoma cells. Mol Cancer Ther 11: 1454-1466, 2012.

12. Iorio MV, Ferracin M, Liu CG, Veronese A, Spizzo R, Sabbioni S, Magri E, Pedriali M, Fabbri M, Campiglio M, et al: MicroRNA gene expression deregulation in human breast cancer. Cancer Res 65: 7065-7070, 2005

13. Heller G, Weinzierl M, Noll C, Babinsky V, Ziegler B, Altenberger C, Minichsdorfer C, Lang G, Döme B, End-Pfützenreuter A, et al: Genome-wide miRNA expression profiling identifies $m i R-9-3$ and $m i R-193 a$ as targets for DNA methylation in non-small cell lung cancers. Clin Cancer Res 18: 1619-1629, 2012.

14. Wang LQ, Kwong YL, Kho CS, Wong KF, Wong KY, Ferracin M, Calin GA and Chim CS: Epigenetic inactivation of $m i R-9$ family microRNAs in chronic lymphocytic leukemia - implications on constitutive activation of $\mathrm{NF}_{\kappa} \mathrm{B}$ pathway. Mol Cancer 12: 173, 2013.

15. Zhu W, Shan X, Wang T, Shu Y and Liu P: miR-181b modulates multidrug resistance by targeting BCL2 in human cancer cell lines. Int J Cancer 127: 2520-2529, 2010.

16. Rao X, Di Leva G, Li M, Fang F, Devlin C, Hartman-Frey C, Burow ME, Ivan $M$, Croce $C M$ and Nephew KP: MicroRNA-221/222 confers breast cancer fulvestrant resistance by regulating multiple signaling pathways. Oncogene 30: 1082-1097, 2011.

17. Yang H, Kong W, He L, Zhao JJ, O'Donnell JD, Wang J, Wenham RM, Coppola D, Kruk PA, Nicosia SV, et al: MicroRNA expression profiling in human ovarian cancer: $m i R$-214 induces cell survival and cisplatin resistance by targeting PTEN. Cancer Res 68: 425-433, 2008.

18. Zhou M,Zeng J, Wang X, Guo Q, Huang T, Shen H, Fu Y, Wang L, Jia $J$ and Chen C: MiR-370 sensitizes chronic myeloid leukemia K562 cells to homoharringtonine by targeting Forkhead box M1. J Transl Med 11: 265, 2013.

19. Weng H, Huang H, Dong B, Zhao P, Zhou H and Qu L: Inhibition of miR-17 and miR-20a by oridonin triggers apoptosis and reverses chemoresistance by derepressing BIM-S. Cancer Res 74: 4409-4419, 2014

20. Nishioka C, Ikezoe T, Yang J, Nobumoto A, Tsuda $M$ and Yokoyama A: Downregulation of miR-217 correlates with resistance of $\mathrm{Ph}^{+}$leukemia cells to $\mathrm{ABL}$ tyrosine kinase inhibitors. Cancer Sci 105: 297-307, 2014.

21. Wang WZ, Pu QH, Lin XH, Liu MY, Wu LR, Wu QQ, Chen YH, Liao FF, Zhu JY and Jin XB: Silencing of miR-21 sensitizes CML CD34 ${ }^{+}$stem/progenitor cells to imatinib-induced apoptosis by blocking PI3K/AKT pathway. Leuk Res 39: 1117-1124, 2015.

22. Sun C, Li N, Yang Z, Zhou B, He Y, Weng D, Fang Y, Wu P, Chen $\mathrm{P}$, Yang X, et al: miR-9 regulation of BRCA1 and ovarian cancer sensitivity to cisplatin and PARP inhibition. J Natl Cancer Inst 105: 1750-1758, 2013.

23. Wang H, Zhang W, Zuo Y, Ding M, Ke C, Yan R, Zhan H, Liu J and Wang J: miR-9 promotes cell proliferation and inhibits apoptosis by targeting LASS2 in bladder cancer. Tumour Biol 36: 9631-9640, 2015

24. Kovalchuk O, Filkowski J, Meservy J, Ilnytskyy Y, Tryndyak VP, Chekhun VF and Pogribny IP: Involvement of microRNA-451 in resistance of the MCF-7 breast cancer cells to chemotherapeutic drug doxorubicin. Mol Cancer Ther 7: 2152-2159, 2008.

25. Zhu H, Wu H, Liu X, Evans BR, Medina DJ, Liu CG and Yang JM: Role of MicroRNA miR-27a and miR-451 in the regulation of $M D R 1 / \mathrm{P}$-glycoprotein expression in human cancer cells. Biochem Pharmacol 76: 582-588, 2008.

26. Feng DD, Zhang H, Zhang P, Zheng YS, Zhang XJ, Han BW, Luo XQ, Xu L, Zhou H, Qu LH, et al: Down-regulated miR-331-5p and miR-27a are associated with chemotherapy resistance and relapse in leukaemia. J Cell Mol Med 15: 2164-2175, 2011. 